\title{
多孔碳材料的设计合成及其在能源存储与转换领域中 的应用
}

\author{
刘明贤，缪灵，陆文静，朱大章，徐子颉，甘礼华*，陈龙武 \\ 同济大学化学科学与工程学院, 上海市化学品分析、风险评估与控制重点实验室, 上海 200092 \\ * 联系人, E-mail: ganlh@ tongji.edu.cn
}

2016-09-26 收稿, 2017-01-03 修回, 2017-01-10 接受, 2017-01-18 网络版发表

国家自然科学基金(21207099, 21273162, 21473122)和上海市科学技术委员会项目(14DZ2261100)资助

\begin{abstract}
摘要人类当前面临越来越突出的能源短缺和环境恶化两大难题, 新能源的开发具有极其重要意义. 超级电容 器是实现能源存储与转换的一种新兴绿色储能器件, 具有非常广阔的应用前景. 电极材料是储能器件的关键部件, 而比表面积、孔结构、电导率和表面性质是决定其电化学性能的4个关键因素，上述因素通常又依赖于其合成方法 和条件. 多孔碳材料具有成本低廉、比表面积与电导率高、微结构可控/表面易于功能化以及优越的化学稳定性和 突出的离子可及性等特点, 通过合成方法和条件的调控, 设计合成的多孔碳作为储能材料使用时展现出高的能量 密度与功率密度, 以及优越的电化学循环稳定性能. 本文首先介绍目前活性碳、碳气凝胶、碳纤维、介孔碳、碳 纳米管和石墨烯等多种形态的碳材料的研究进展; 然后结合本研究组的研究工作, 对分级孔碳、多孔碳球、超微 孔碳、功能化多孔碳以及多孔碳复合材料的设计合成及其在能源存储与转换领域中的应用研究状况进行总结; 最 后对其发展趋势作出适当的评述.
\end{abstract}

关键词多孔碳, 合成, 储能材料, 超级电容器, 电化学性能

随着经济的不断发展，不可再生的化石能源的 大量消耗, 能源短缺和环境恶化已经成为人类面临 的越来越突出的两大难题, 新能源的开发和节能减 排成为当今世界的一个重要课题 ${ }^{[1]}$. 当前用于汽车驱 动的动力电池主要是镍氢电池和锂离子电池, 但其 技术性能、使用的稳定性、安全性和生产成本等问题 仍有待于解决, 至今为止, 动力电池仍存在充电时间 长、续驶里程短、电池成本高等问题. 电化学电容器 又称为超级电容器和超大容量电容器, 是随着材料 科学的突破而出现的一种新型功率型储能器件, 1957 年Becker首次提出电化学电容器的概念, 1978年 Panasonic 公司将其实现了商业化 ${ }^{[2]}$. 与锂离子电池 相比, 超级电容器具有功率密度很高、瞬间释放大电
流、充电时间极短、充电效率高、对环境无污染、使 用寿命长和无记忆效应等优异特性，最有希望成为 一种新型的绿色能源 ${ }^{[3]}$.

根据电能存储机制的不同, 超级电容器可分为 两种主要类型, 即双电层电容器(EDLC) 和噟电容器. 噟电容器利用电极上的活性材料与电解液中的离子 发生氧化-还原反应, 通过跨越电解质与电极间界面 的可逆法拉第电荷转移产生赝电容, 赝电容器的行为 更像锂离子电池. 过渡金属氧化物(如 $\mathrm{MnO}_{2}{ }^{[4]}, \mathrm{NiO}^{[5]}$, $\mathrm{RuO}_{2}{ }^{[6]}, \mathrm{V}_{2} \mathrm{O}_{5}{ }^{[7]}$ 和导电聚合物(如聚噻吩, 聚苯胺, 聚 吡咯及其衍生物) 已得到了人们的广泛研究 ${ }^{[8,9]}$. EDLC类似于传统的物理电容器，它们都依靠电荷分 离来存储能量, 所不同的是, 在EDLC中电荷的分离 
过程发生在负极和正极的界面上, 并形成 2 个双电层, 每层相当于一个传统的电容器, 因而EDLC的比电容 比传统电容器要高几个数量级. 由于在EDLC中电荷 分离发生在电极和电解质界面内的更小距离间, 它 只涉及到界面上的离子运动, 因此, EDLC的电容量 取决于电极的表面积, 碳材料包括活性碳、碳气凝 胶、碳纤维、介孔碳、碳纳米管和石墨烯都具有高的 比表面积和快速电荷传输性能, 是一种很有前途的 高性能超级电容器电极材料 ${ }^{[10]}$.

碳基材料是近年来超级电容器中应用最多的电 极材料 ${ }^{[11]}$, 目前以活性碳为电极材料的超级电容器 已经实现了商业化, 但活性碳基超级电容器还无法 满足脉冲大电流充放的需求. 为了扩大活性碳的孔 径和比表面积, 提高其比电容, 有关活性碳表面改 性、超高比表面积的活性碳和介孔活性碳的制备及其 作为电极材料的研究层出不穷, 但仍未取得令人惊 喜的结果. 碳气凝胶是一种由纳米碳颗粒构成的纳 米多孔碳材料, 具有连续网络孔洞、比表面积大、电 导率较高等特点, 美国劳伦兹利物莫尔国家实验室 首先开展碳气凝胶基超级电容器的研究, 这种超级 电容器具有良好的循环性能和优于活性碳基超级电 容器的比电容 ${ }^{[12,13]}$. 从碳基超级电容器储能原理来 看, 碳纳米管算是一种有前途的碳基超级电容器电 极材料 ${ }^{[14]}$, 因为单壁碳纳米管和多壁碳纳米管都集 高比表面积、高导电性、机械稳定性和电催化活性于 一体, 用于超级电容器时电解质溶液更容易渗透活 性颗粒, 由碳纳米管形成的多孔网络可以使离子更 快地扩散到活性组分的表面, 还能缓冲充放电过程 中的体积变化, 提高电化学循环性能, 因此, 碳纳米 管作为超级电容器电极材料已被广泛研究. 目前提 高碳纳米管基超级电容器的比电容主要有3种方法, 即引人活性基团和缺陷对碳纳米管进行功能化, 以 提供其噟电容, 将碳纳米管与其他碳材料结合形成 多级孔结构, 或者将它与金属氧化物和导电聚合物 结合引人噟电容 ${ }^{[15]}$. 石墨烯也具有突出的电学、机械 和电化学性能, 因此已经被用于高性能超级电容器 的制作. 目前人们已经发展出机械剥离、氧化还原、 $\mathrm{SiC}$ 外延生长和化学气相沉积(CVD) 等制备高度分散 石墨烯薄片的方法, 并将制备条件对石墨烯的电化 学电容性能的影响进行广泛研究; 为了进一步提高 比电容, 将石墨烯制成不同的多孔材料也有许多报 道. 近年来, 人们还发展出另一种有效提高石墨烯电
极的比电容的策略, 即通过在氧化石墨烯间引人诸 如表面活性剂类的隔离剂或稳定剂, 以防止石墨烯 之间发生团聚，其中的隔离剂不仅可以改善电解液 的易接近性，还可以提高石墨烯片纳米通道及表面 的电化学利用率. 与碳纳米管一样, 作为电极材料使 用时, 石墨烯也表现出相对低的比电容, 而在石墨烯 中引人具有高比电容的另一相电活性物, 将其制备 成石墨烯/导电聚合物复合电极及石墨烯/金属氧化物 杂化电极, 则有望获得高性能超级电容器, 目前石墨 烯基复合电极材料已得到了广泛的研究 ${ }^{[16 ~ 18]}$. 介孔 碳是一种典型的多孔碳材料, 具有大的孔道和高的 比表面积, 有利于离子的迁移与电荷积累, 这就预示 着其在锂离子电池和双电层电容器方面将有着一定 的应用潜力. 有有序介孔碳 $(\mathrm{OMC})$ 作为超级电容器 电极材料也被广泛研究, OMC通常由有序介孔二氧 化硅为硬模板或者嵌段聚合物为软模板, 采用不同 的碳源制备, 它具有高的比表面积、高孔隙率和孔径 尺寸在一定范围内可调的特点, 研究者在如何提高 介孔碳的孔体积和比表面积等方面作出了巨大的努 力, 然而介孔碳的比电容与其比表面积并不成线性 关系. 通过多方努力, 介孔碳的电化学性能得到了适 度的提高, 例如, 在 $1 \mathrm{~mA} \mathrm{~cm}$ 的低电流密度下, 典 型的有序介孔碳在有机电解液和水系电解液中的质 量比电容分别达到100 120和150 200 $\mathrm{F} \mathrm{g}^{-1}$. 由此可 知, 有序介孔碳的电化学电容性能并不好, 将有序介 孔碳制备成为功能化 $\mathrm{OMC}$ 电极、 $\mathrm{OMC}$ 基复合电极或 杂化电极的研究仍屡见报道 ${ }^{[19,20]}$. 近几年随着可穿 戴电子器件的飞速发展, 高度柔性能量转换或储存 器件备受人们的关注, 柔性、轻便、可集成、可穿戴 的纤维状超级电容器应运而生. 与需要无氧、无水苛 刻条件的染料敏化太阳能电池、聚合物太阳能电池和 锂离子电池不同, 在温和的空气气氛中, 纤维状超级 电容器很容易直接通过扭曲 2 个纤维电极, 或者将 2 个电极缠绕在纤维基体上形成同轴结构而制造, 它 们运行更加稳定, 使用寿命更长. 例如, 可以将涂覆 凝胶电解质的两根定向排列的碳纳米管纤维扭曲成 集碳纳米管纤维的高导电性和机械强度以及柔软性 于一体的纤维状超级电容器 ${ }^{[21]}$, 最近, 同济大学陈 涛课题组 ${ }^{[22]}$ 将高度取向的碳纳米管与较大赝电容的 二硫化钼纳米片相结合, 制备致密结构的复合材料, 以其为电极获得了可拉伸度高达 $240 \%$ 的柔性可拉伸 超级电容器, 该柔性可拉伸的纤维状超级电容器展 
现出高达 $13.16 \mathrm{~F} \mathrm{~cm}^{-3}$ 的体积比电容和 10000 次循环 后电容保持率为 $98 \%$ 的超高循环稳定性能. 此外, 轻 质、柔性、高强度和导电性的石墨烯纤维也被用于制 备具有典型双电层电容行为的纤维状超级电容器, 其 中的多孔和褶皱结构的石墨烯纤维是由CVD法合成 的石墨烯通过组装而获得的 ${ }^{[23]}$, 两根石墨烯纤维被扭 曲制成一种基于凝胶电解质的全固态纤维状超级电容 器, 其比电容达到了 $2.13 \mathrm{mF} \mathrm{cm}^{-2}$.

综上所述, 电极材料是超级电容器的关键部件, 高性能碳基电极材料的制备仍然是大容量高比功率 超级电容器研制的关键科技问题. 目前以活性碳、碳 气凝胶、碳纳米管、介孔碳、石墨烯、洋苟状碳 ${ }^{[24]}$ 、 衍生碳 ${ }^{[25]}$ 和碳纳米笼 ${ }^{[26]}$ 作为超级电容器电极材料已 有了大量研究, 虽然这些碳材料各有其优点, 相关研 究竞相开展, 但仍未取得突破性进展. 从储能原理来 看, 对于超级电容器电极材料, 比表面积、孔结构、 电导率和表面性质是决定其比电容、功率密度和能量 密度等电化学性能的4个关键因素, 而上述因素通常 又依赖于其制备方法和条件 ${ }^{[27]}$. 与碳纳米管、石墨 烯、有序介孔碳以及洋葱状碳、碳纳米笼等特殊形态 碳材料相比, 多孔碳材料除了具有高电导性、良好化 学稳定性和热稳定性等之外, 它的最大优点在于其 成本低廉, 通过碳源前驱体的选择以及合成方法和 条件的篮选, 可以实现对多孔碳材料的结构控制, 设 计合成出孔洞网络相互贯通、孔分布合理和表面性能 可调的多孔碳材料, 还能够缩短离子扩散路径和缓 冲其在充放电中的体积变化, 从而提高其作为超级 电容器电极材料的能量密度、倍率性能和电化学循环 稳定性能. 正因为如此, 近年来本研究组成员在多孔 碳材料的设计合成及其在能源存储与转换领域中的 应用作出了相当大的努力, 本文将结合本研究组在 这方面开展的研究工作, 对分级孔碳、多孔碳球、超 微孔碳、功能化多孔碳和多孔碳复合材料等研究状况 进行总结, 并对其发展趋势作出适当的评述.

\section{1 多孔碳的合成与作为储能材料的应用}

\section{1 分级孔碳}

分级孔碳又称多级孔碳, 通常是指具有微孔、介 孔和/或大孔的多级孔道的碳材料, 它具有良好的导 电性、高比表面积和稳定性好的特点, 在锂离子电 池、超级电容器、太阳能电池、燃料电池、储氢、气
体分离和催化剂载体等方面都有着不凡的应用前

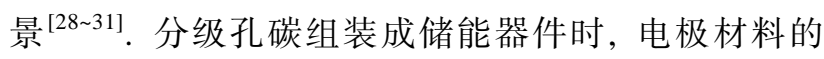
有效电化学接触面积大、扩散距离短、大倍率传输效 率高, 因而往往表现出优异的储锂及储能容量, 尤其 是其高传质速率带来的高倍率性能, 使其成为近年 来的研究热点 ${ }^{[32 \sim 34]}$.

分级孔碳一般通过模板法或模板-活化相结合的 方法而制备. 模板法的最大优点在于碳基体反相复 制模板结构，通过改变模板剂的结构和用量可以有 效调节产物的孔结构. 双模板(软硬模板)法是目前最 常用也是报道最多的制备方法, 它通常利用硅溶胶 等硬模板与表面活性剂和嵌段共聚物等软模板的共 同作用调控生物质或聚合物的微结构，从而制备不 同织构的分级孔碳材料. 然而大多数模板剂不仅价 格昂贵, 还会对环境造成危害, 同时又必须经历一系 列去除模板以形成分级孔结构的后处理步骤, 这些 都严重阻碍了双模板法发展的步伐. Qie等人 ${ }^{[34]}$ 通过 化学活化法对聚吡咯薄片进行结构优化制备了功能 化三维分级孔碳材料(THPCs), THPCs的比表面积高 达 $2870 \mathrm{~m}^{2} \mathrm{~g}^{-1}$, 孔径分布在 $0.68,1.26,2.73$ 和 $5 ~ 50 \mathrm{~nm}$ 之间; 作为超级电容器电极材料, 在电流密度为 5.0 $\mathrm{A} \mathrm{g}^{-1}$ 时的比电容为 $200.8 \mathrm{~F} \mathrm{~g}^{-1}$, 经过 10000 次循环后 的比电容保持率仍高达 $95.8 \%$. 本研究组 ${ }^{[35]}$ 利用溶剂 蒸发自组装的方法, 通过控制三嵌段共聚物、正硅酸 乙酯和间苯三酚-对苯二甲醛三组分聚合体系中对苯 二甲醛与间苯三酚的摩尔比, 分步碳化以及去除碳硅复合物中的二氧化硅等步骤, 合成出高比表面的 双峰互联有序微-介孔碳(OMMC). OMMC具有约 9.0 $\mathrm{nm}$ 的初级介孔和约 $3.0 \mathrm{~nm}$ 的次生介孔, 以及约 1.3 $\mathrm{nm}$ 的初级微孔和 0.7 0.6 $\mathrm{nm}$ 的次生微孔. Zhang等 人 $^{[36]}$ 以葡萄糖和果糖为碳源, 聚离子液体为结构稳 定剂和造孔剂, 合成了粒子尺寸小于 $50 \mathrm{~nm}$ 的分级孔 碳纳米粒子. Qian等人 ${ }^{[37]}$ 将金属有机骨架嵌人酚醛树 脂中, 以优化分级孔碳材料的孔结构, 这种多级孔道 结构使其对二氧化碳的捕获量大幅度增加. 这些都 为无模板制备分级孔碳材料提供了思路, 而更为绿 色的无模板合成方法则是致力于以生物聚合物为碳 源制备不同结构形态的分级孔碳材料 ${ }^{[38]}$.

迄今为止，文献报道的基于生物质的分级孔碳 的合成方法, 基本上限制在将生物质碳源进行先高 温碳化后化学活化, 这种方法对生物聚合物基碳材 料的微结构的调控及优化是十分有限的, 因而无法 
满足分级孔碳材料的可控合成等多元要求. 本课题 组 ${ }^{[39]}$ 制备了具有大量生物聚合物微细孔结构和丰富 毛孔表面官能团的廉价香蕉皮构筑的分级孔碳泡沫. 通过香蕉皮表面的羧基和羟基与锌离子络合, 形成 类似金属有机骨架聚合物的含锌配合物, 以基于香 蕉皮的锌配合物为模板, 进一步结合额外的碳源如 氨基酚-糠醛树脂聚合物, 合成了酚醛树脂-香蕉皮含 锌配位聚合物的复合物, 将上述复合物直接碳化获 得了分级孔碳泡沫(HPCFs), 由香蕉皮构筑合成分级 孔碳泡沫过程如图 1所示. 所得 HPCFs 具有三维互通 的大孔核心以及孔壁上的介孔通道和微孔孔隙, 其 比表面积高达 $1650 \mathrm{~m}^{2} \mathrm{~g}^{-1}$, 孔体积为 $1.26 \mathrm{~cm}^{3}$, 平均 孔径为 $3.01 \mathrm{~nm}$, 孔径主要分布在 $1.8,2.8,3.6$ 和 16.4 $\mathrm{nm}$ 处. 作为超级电容器电极材料, HPCFs电极表现出 优秀的电化学电容性能和高倍率特性, 在电流密度 为 $1.0 \mathrm{~A} \mathrm{~g}^{-1}$ 时其比电容为 $206 \mathrm{~F} \mathrm{~g}^{-1}$, 在电流密度为 10 $\mathrm{A} \mathrm{g}^{-1}$ 时其比电容仍保持在 $182 \mathrm{~F} \mathrm{~g}^{-1}$. 由于其易用性、 生产成本低、电化学性能优越, 这类基于香蕉皮复合
物合成的分级孔碳泡沫在超级电容器、气体吸附等领 域都有着广泛的应用前景.

\section{2 多孔碳球}

与其他多孔碳材料相比，近期研究者更加关注 拥有一定特殊规则的微观形貌的多孔材料如管、球或 类似石墨烯的二维有序结构. 其中, 碳球因其具有规 则的球形结构、单分散性好、微球球径可调、比表面 积高等特点, 在吸附、催化、锂离子电池和超级电容 器电极材料等领域有着重要的应用前景 ${ }^{[40 \sim 44]}$. 此外, 球形结构在堆积时所形成的大孔、球与球之间的自由 滑动性以及碳微球的良好导电性使得碳微球作为超 级电容器电极材料时能够形成丰富的双电层结构, 展现出优秀的电化学性能.

长期以来, 研究工作主要集中在碳微球和碳纳 米球的制备及应用. 例如, Choma等人 ${ }^{[45]}$ 总结了使用 延伸的Stöber法制备微孔、介孔或微-介孔碳球的方 法. 文中所报道的方法包括使用二氧化硅等胶体粒
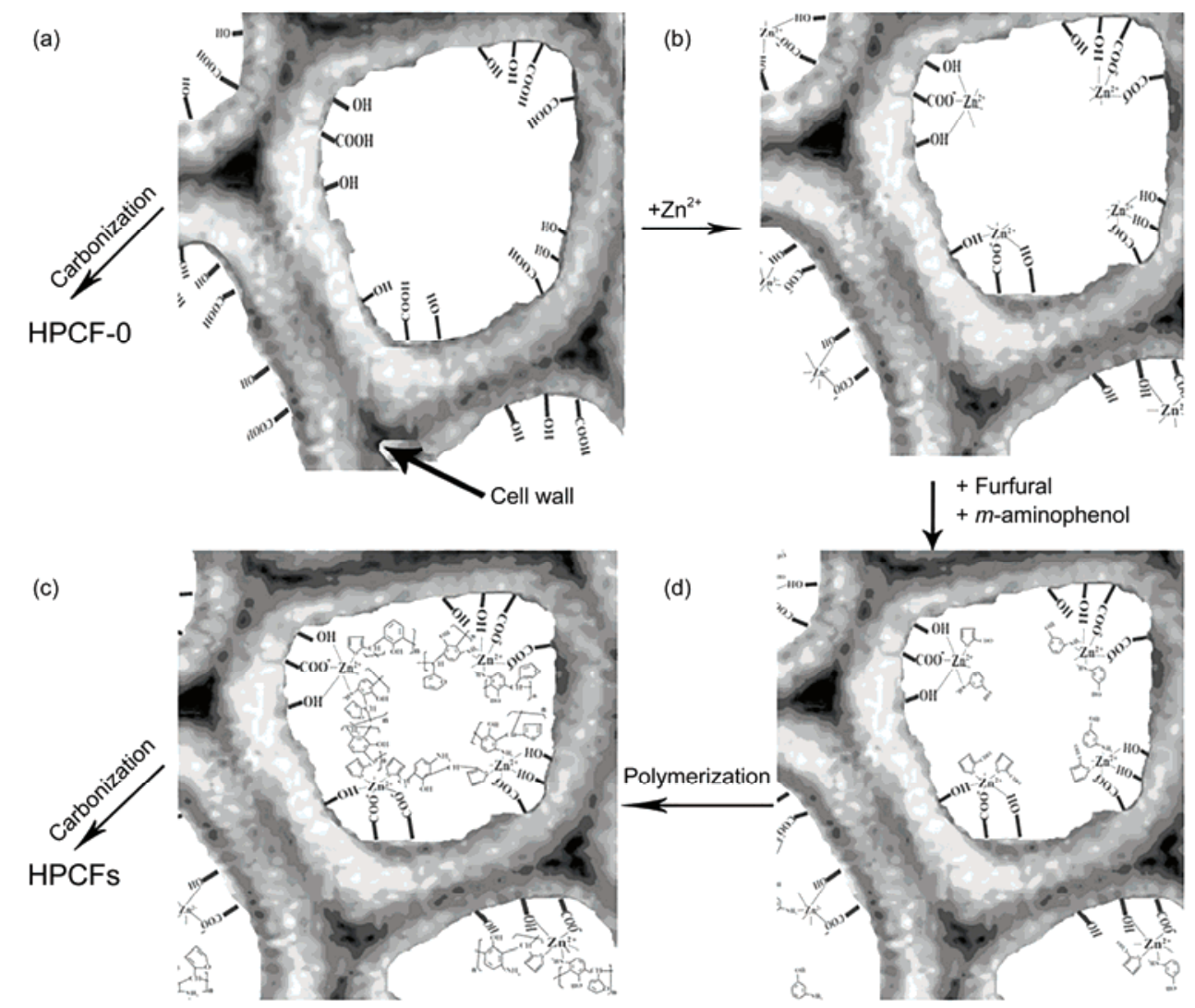

图 1 由香蕉皮构筑分级孔碳泡沫HPCFs的合成过程示意图 ${ }^{[39]}$

Figure 1 Schematic illustration of the formation process of $\mathrm{HPCFs}^{[39]}$ 
子、表面活性剂和嵌段共聚物作为模板剂制备多孔碳 球. $\mathrm{Xu}$ 和 $\mathrm{GuO}^{[46]}$ 在水溶液中以苯酚为前驱体和六亚甲 基四胺为固化剂制备得到了单分散好、粒径为300 $1000 \mathrm{~nm}$ 的酚醛树脂纳米球. 随着苯酚/HMTA的摩尔 比的增加, 制备的碳纳米球的球径减小, 即六亚甲基 四胺的用量越大, 所得碳纳米球的球径越大. 而 Choma等人 ${ }^{[47]}$ 则以间苯二酚和甲醛作为碳前驱体, 氨水作催化剂, 利用二氧化硅制造介孔制备了单分 散好、粒径为120 500 $\mathrm{nm}$ 的介孔碳球, 其比表面积为 $682 \mathrm{~m}^{2} \mathrm{~g}^{-1}$, 孔体积达到 $0.65 \mathrm{~cm}^{3} \mathrm{~g}^{-1}$. Fang等人 ${ }^{[48]}$ 报 道了一种新的低浓度水热方法合成大小均一、高度有 序体心立方结构的介孔球状碳纳米粒子. 该方法以 嵌段共聚物F127作模板, 酚醛树脂为碳源, 通过调 节反应物浓度制得了球径在20 140 nm的有序介孔碳 球. 研究表明, 当反应体系中苯酚与水的摩尔比为 $1: 250$ 时, 制得的碳球的比表面积为 $1131 \mathrm{~m}^{2} \mathrm{~g}^{-1}$, 孔 体积为 $1.52 \mathrm{~cm}^{3} \mathrm{~g}^{-1}$, 孔径集中在 $2.6 \mathrm{~nm}$. Qiao等人 ${ }^{[49]}$ 使用酚醛树脂为聚合物前驱体, 硅酸盐低聚物为无 机前驱体, 十六烷基六甲基氯化铵为模板制备介孔 碳纳米球. 所得的碳纳米球尺寸均一, 球径为 180 $850 \mathrm{~nm}$, 且球径可以通过乙醇用量得到调控. 乙醇与 水的体积比为 $1: 4.75$ 时, 球径 $180 \mathrm{~nm}$; 乙醇与水的体 积比为 $1: 2.38$ 时, 球径增大至 $800 \mathrm{~nm}$. Li等人 ${ }^{[50]}$ 采用 酚醛树脂为碳源, 表面活性剂和嵌段共聚物F127为 软模板, 二氧化硅泡沫球为硬模板, 制备出球径在 3 5 $\mu \mathrm{m}$, 孔径分布在3.5 60 nm范围内的分级孔结构 碳球. 该分级孔碳球的比表面积为 $1320 \mathrm{~m}^{2} \mathrm{~g}^{-1}$, 孔体 积高达 $3.5 \mathrm{~cm}^{3} \mathrm{~g}^{-1}$. 当作为超级电容器电极材料, 在 $\left(\mathrm{C}_{2} \mathrm{H}_{5}\right)_{4} \mathrm{NBF}_{4} / \mathrm{C}_{3} \mathrm{H}_{6}$ 电解液中, 电流密度为 $0.5 \mathrm{~A} \mathrm{~g}^{-1}$ 时 的比电容为 $97 \mathrm{~F} \mathrm{~g}^{-1}$; 在 $0.2 \mathrm{~mol} \mathrm{~L} \mathrm{H}_{2}^{-1} \mathrm{SO}_{4}$ 电解液中, 电流密度为 $0.5 \mathrm{~A} \mathrm{~g}^{-1}$ 时的比电容高达 $208 \mathrm{~F} \mathrm{~g}^{-1}$; 电流 密度为 $30 \mathrm{~A} \mathrm{~g}^{-1}$ 时的比电容达到 $146 \mathrm{~F} \mathrm{~g}^{-1}$.

本课题组 ${ }^{[51]}$ 发展了一种新型的水热-乳液-活化 的方法, 制备出粒径为 $0.5 \sim 2.0 \mu \mathrm{m}$, 比表面积为 $449 \sim$ $1212 \mathrm{~m}^{2} \mathrm{~g}^{-1}$, 孔径在 2.6 4.0 $\mathrm{nm}$ 的介孔碳微球(图 2(a)). 将介孔碳微球制成超级电容器电极材料, 在不 同电流密度下的恒流充放电曲线如图2(b) 所示, 以6 $\mathrm{mol} \mathrm{L} \mathrm{L}^{-1} \mathrm{KOH}$ 溶液为电解液时, 该电极在电流密度为 $10 \mathrm{~A} \mathrm{~g}^{-1}$ 时的比电容达 $157 \mathrm{~F} \mathrm{~g}^{-1}$. 此外, 本课题组 ${ }^{[52]}$ 还提出了一种新型“种子”法制备多孔碳纳米球. 该 方法以间苯三酚和对苯二甲醛为聚合前驱体, 通过 控制反应时间获得粒径约为 $35 \mathrm{~nm}$ 的胶体粒子为“种
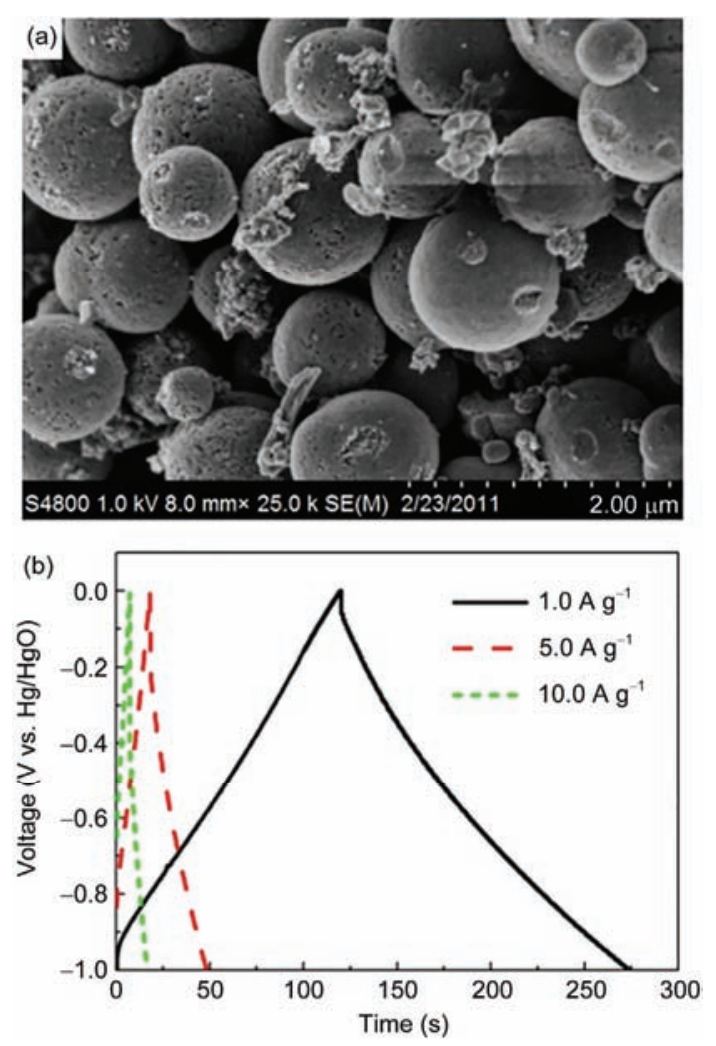

图 2 (网络版彩色)介孔碳微球的扫描电子显微镜(SEM)(a)及其在不 同电流密度下的恒流充放电曲线 $(b)^{[51]}$

Figure 2 (Color online) SEM images (a) and GCD curves (b) at different current densities of mesoporous carbon nanospheres ${ }^{[51]}$

子”，在氨水的催化作用下，间苯二酚和甲醛在“种 子”表面进行聚合反应，经碳化、活化合成出粒径约 为 $90 \mathrm{~nm}$, 比表面积为 $2123 \mathrm{~m}^{2} \mathrm{~g}^{-1}$, 孔体积为 $2.47 \mathrm{~cm}^{3}$ $\mathrm{g}^{-1}$ 的多孔碳纳米球. 该多孔碳纳米球作超级电容器 电极, 以 $6 \mathrm{~mol} \mathrm{~L}^{-1} \mathrm{KOH}$ 为电解液, 在电流密度为 10 $\mathrm{A} \mathrm{g}^{-1}$ 时的比电容达到了 $238 \mathrm{~F} \mathrm{~g}^{-1}$. 这种“种子”策略还 可以用来制备其他一些中空或是复合结构的聚合物、 碳、硅等纳米材料, 具有一定的普适性. 本课题组 ${ }^{[53]}$ 还以间苯二酚-甲醛合成酚醛树脂预聚物为前驱体, 在氨水作为催化剂的条件下加人硅溶胶, 通过聚合、 高温碳化、去模板, 再经过化学活化制得了粒径约为 $500 \mathrm{~nm}$ 的微孔-介孔碳球(MMCSs)(图3), 典型的 MMCSs的介孔孔径为 $11.0 \mathrm{~nm}$, 同时出现 $2 \mathrm{~nm}$ 以下的 微孔, MMCSs的总孔体积高达 $1.04 \mathrm{~cm}^{3} \mathrm{~g}^{-1}$, 其中的 $0.63 \mathrm{~cm}^{3} \mathrm{~g}^{-1}$ 为介孔体积, 比表面积高达 $1620 \mathrm{~m}^{2} \mathrm{~g}^{-1}$, 其中 $847 \mathrm{~m}^{2} \mathrm{~g}^{-1}$ 的贡献来自于微孔, 大量微孔的存在 为电荷的存储提供了巨大的比表面, 因此, 由该碳球 制备的电极在 $6 \mathrm{~mol} \mathrm{~L}^{-1} \mathrm{KOH}$ 电解液中可以表现出优 

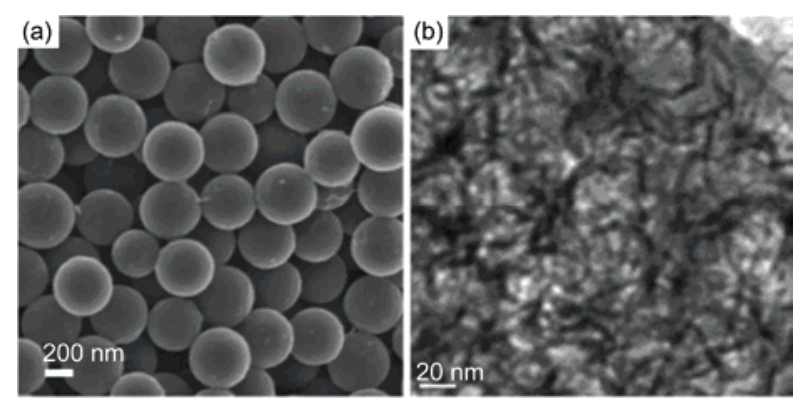

图 3 微孔-介孔碳球SEM(a) 和透射电子扫描显微镜(TEM)(b)图片 ${ }^{[53]}$ Figure 3 SEM (a) and TEM (b) images of micro-mesoporous carbon nanospheres ${ }^{[33]}$

良的电化学性能: 当电流密度为 $0.5 \mathrm{~A} \mathrm{~g}^{-1}$ 时, 比电容 达314 $\mathrm{F} \mathrm{g} \mathrm{g}^{-1}$, 且在 500 次充放电后, 比电容仍保持在 $301 \mathrm{~F} \mathrm{~g}^{-1}$.

本研究组还通过模板法制备得到了孔道大小易 于调控的介孔碳微球 ${ }^{[54]}$. 在乙醇-水-氨水混合溶剂体 系中，通过控制正硅酸乙酯的浓度及其水解-缩聚反
应时间，得到了不同粒径的 $\mathrm{SiO}_{2}$ 纳米粒子. 向体系中 分别加人间苯二酚和甲醛，经过溶胶-凝胶过程、聚 合反应、碳化得到 $\mathrm{SiO}_{2} @ \mathrm{C}$ 微球. 经 $\mathrm{NaOH}$ 蚀刻二氧化 硅模板, 最后获得了粒径大约为 $500 \mathrm{~nm}$, 比表面积为 659 872 $\mathrm{m}^{2} \mathrm{~g}^{-1}$, 孔径为3.2 14 $\mathrm{nm}$ 的介孔碳微球 $(\mathrm{MCMs})$ (图4). 以 $6 \mathrm{~mol} \mathrm{~L}^{-1} \mathrm{KOH}$ 电解液时, $\mathrm{MCMs}$ 电极 材料在电流密度为 $1.0 \mathrm{~A} \mathrm{~g}^{-1}$ 时的比电容达 $289 \mathrm{~F} \mathrm{~g}^{-1}$. 随后, 我们发展“种子”法合成超微孔@微孔碳纳米 球。通过控制间苯三酚和对苯二甲醛的聚合得到小 粒径的表面富含羟基基团的微孔聚合物纳米粒子， 以该微孔聚合物纳米粒子为“核”，利用第二碳源间 苯二酚/甲醛作为壳组分，通过第二碳源与微孔聚合 物纳米粒子“核”之间的氢键作用，结合氨水作用下 的乳液聚合反应制备核壳结构的微孔聚合物纳米球, 经碳化-活化过程制备得到具有核壳结构的超微孔@ 微孔碳纳米球(UMCNs), UMCNs的合成过程如图5(a) 所示 ${ }^{[5]}$. 所得 $U M C N s$ 的粒径为 $52 \sim 74 \mathrm{~nm}$, 比表面积 (a)

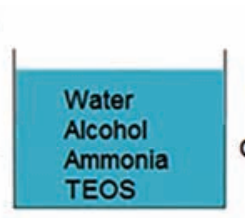

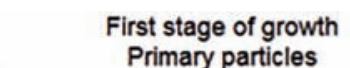

\section{Second stage of growth Third stage of growth}
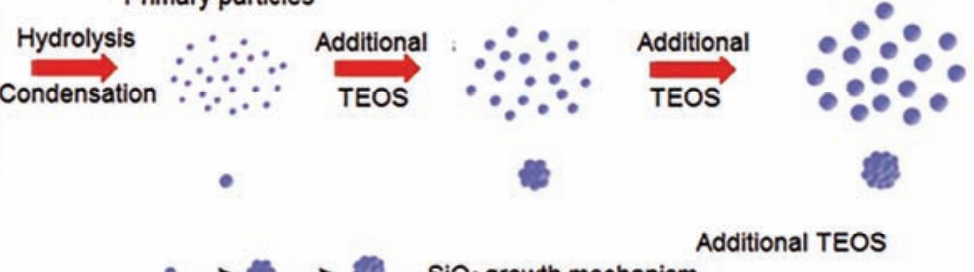

\section{Additional TEOS}

\section{$\rightarrow \rightarrow \mathrm{SiO}_{2}$ growth mechanism}
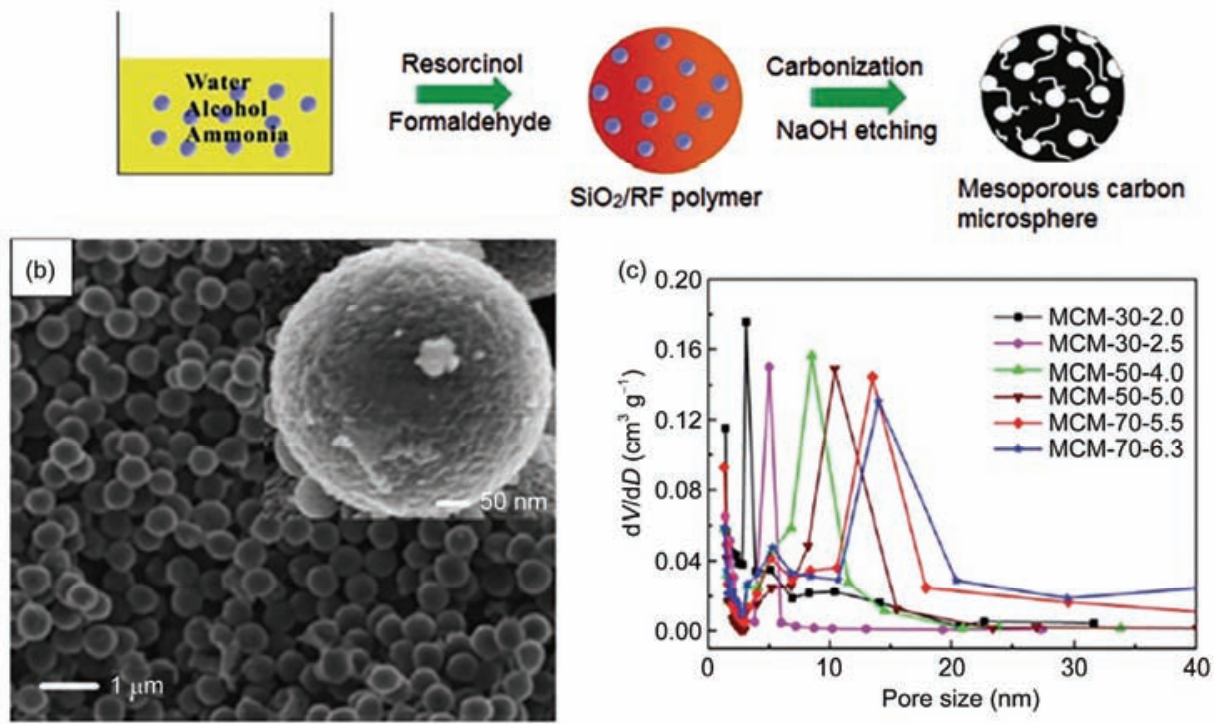

图 4 (网络版彩色)(a) MCMs的合成过程示意图; (b) MCMs的SEM图; (c) MCMs的孔径分布曲线 ${ }^{[54]}$

Figure 4 (Color online) (a) Schematic illustration of the formation process. SEM images (b) and pore size distribution curves (c) of MCMs ${ }^{[54]}$ 

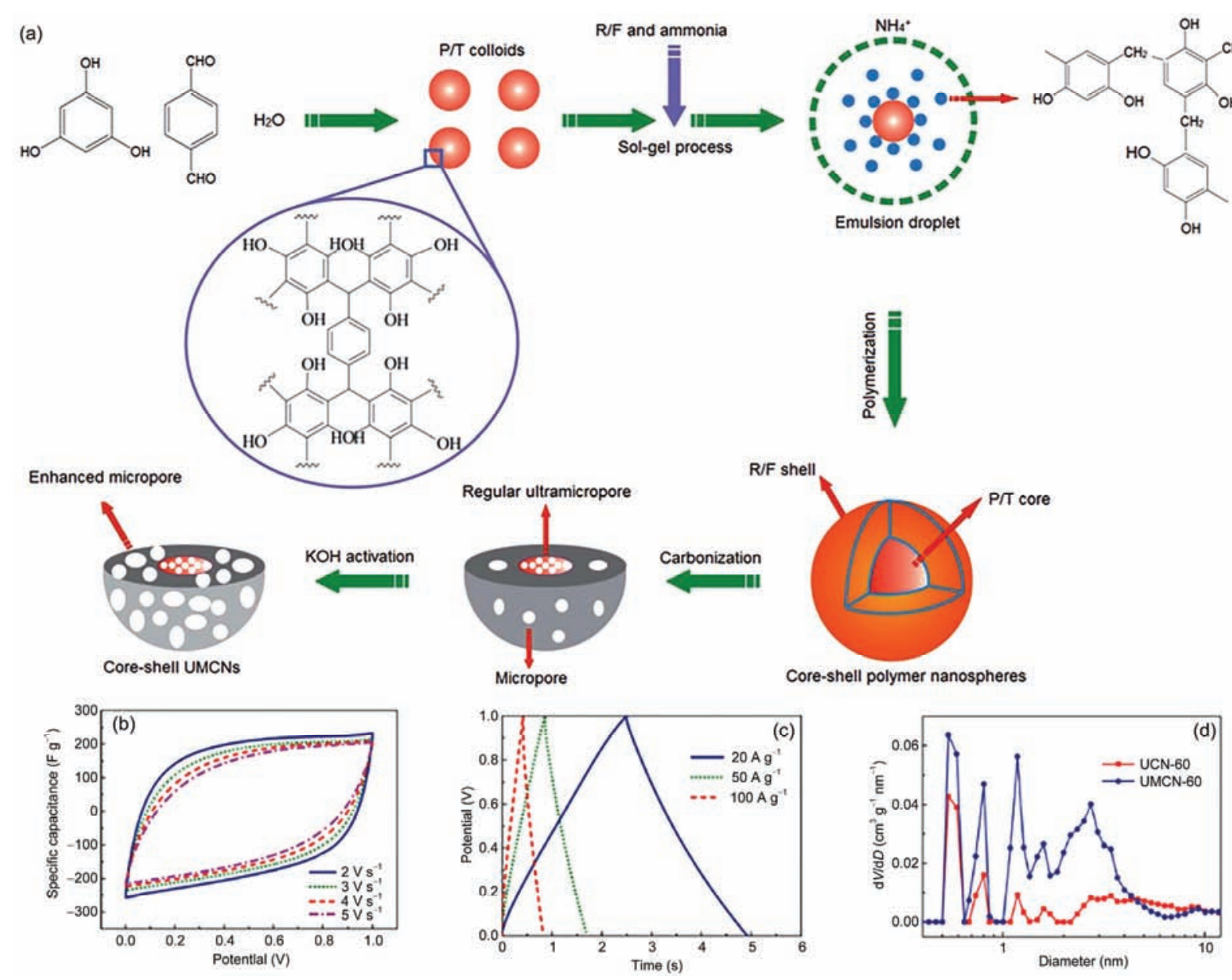

图 5 (网络版彩色) (a) UMCNs的合成过程示意图; (b) UMCNs 在不同扫速下的循环伏安曲线; (c) UMCNs 在不同电流密度下的恒流充放电曲 线; (d) UCNs和UMCNs的孔径分布曲线 $[5]$

Figure 5 (Color online) (a) Schematic illustration of the formation process, (b) CV curves at various scan rates and (c) GCD curves at different current densitiesof UMCNs. (d) Pore size distribution curves of UCNs and UMCNs ${ }^{[55]}$

为 $2156 \mathrm{~m}^{2} \mathrm{~g}^{-1}$, 具有丰富的微孔和介孔孔道(图 $\left.5(\mathrm{~d})\right)$. 研究表明, 当扫描速率从 $1000 \mathrm{mV} \mathrm{s}^{-1}$ 不断增加到 $5000 \mathrm{mV} \mathrm{s}^{-1}$, 由UMCNs碳纳米球制备的电极的循环 伏安曲线仍保持着相当好的对称性和矩形性 (图 5(b)), 表现出极其优异的快速充放电性能. 当电流密 度从 $10 \mathrm{~A} \mathrm{~g}^{-1}$ 增大到 $100 \mathrm{~A} \mathrm{~g}^{-1}$ 时, UMCNs电极的恒流 充放电曲线依然保持着良好的对称性和直线性 (图 5(c)), UMCNs电极展现出超高的倍率性能. 值得指 出的是, 当电流密度为 $1.0 \mathrm{~A} \mathrm{~g}^{-1}$ 时, $\mathrm{UMCNs}$ 电极在 $6 \mathrm{~mol} \mathrm{~L}^{-1} \mathrm{KOH}$ 电解液中的比电容竟然高达 $411 \mathrm{~F} \mathrm{~g}^{-1}$. 一定比例的介孔和大孔可以在电极进行快速充放电 时提供离子迁移的通道, 提高电极的电化学性能. 该 方法为新型纳米结构碳材料在超级电容器中的应用 提供了一种可靠的方法.

\section{3 超微孔碳}

根据国际纯粹与应用化学会(IUPAC)的规定, 可 将多孔材料按照其孔径大小分为微孔 $(<2 \mathrm{~nm})$ 、介孔 $(2 \sim 50 \mathrm{~nm})$ 和大孔(>50 nm) 材料3类, 其中微孔可进一 步分为超微孔 (或亚纳米级孔) $(<0.7 \mathrm{~nm}$ ) 和亚微孔 $(0.7 \sim 2 \mathrm{~nm})$, 因此将直径小于 $0.7 \mathrm{~nm}$ 的微孔材料称为 超微孔材料. 微孔有机聚合物具有密度低、比表面积 大、稳定性高、孔道互通且尺寸均一可控等优点, 还 可以大范围地通过改变有机分子的官能团和采用不 同的合成手段实现对微孔有机聚合物的功能化调 节 ${ }^{[56 ~ 61]}$, 并将其应用于超微孔材料的制备中. 近年 来, 已有文献报道了亚纳米级孔对超级电容器电化 学性能的影响, 结果表明孔径小于 $1 \mathrm{~nm}$ 的微孔可以显 著提高电极材料的比电容, 尤其是直径在 $0.5 \sim 1 \mathrm{~nm}$ 的 
微孔可以被水系电解质离子所利用 ${ }^{[62 ~ 65]}$. 因此, 孔 径小于 $0.7 \mathrm{~nm}$ 的超微孔碳材料作为超级电容器电极 材料有着良好的应用前景. $\mathrm{Xu}$ 等人 ${ }^{[66]}$ 采用聚偏二氟乙 烯为前驱体, 制备一种孔径为 $0.55 \mathrm{~nm}$ 的超微孔材料. 作为超级电容器电极材料, 在 $1.0 \mathrm{~A} \mathrm{~g}^{-1}$ 电流密度下的 比电容为 $194 \mathrm{~F} \mathrm{~g}^{-1}$; 在电流密度增加至 $10 \mathrm{~A} \mathrm{~g}^{-1}$ 时其比 电容仍有 $145 \mathrm{~F} \mathrm{~g}^{-1}$, 表明具有规则孔径的超微孔碳 材料是一种新型的极具应用潜力的超级电容器电极 材料.

本课题组 ${ }^{[67]}$ 以间苯三酚和对苯二甲醛为单体, 在溶剂热条件下制备微孔有机聚合物, 并以该聚合 物为前驱体, 经高温碳化制得一系列新型的超微孔 碳微球(UCMs) 和超微孔碳纳米粒子(UCNs), 超微孔 碳的合成过程示意图如图6(a)所示. 典型样品UCN0.15-220-850由粒径约为 $30 \mathrm{~nm}$ 的碳纳米粒子构成(图 6(b)), 其比表面积为 $842 \mathrm{~m}^{2} \mathrm{~g}^{-1}$, 孔体积为 $0.74 \mathrm{~cm}^{3} \mathrm{~g}^{-1}$, 且具有规则的超微孔 $(0.54 \mathrm{~nm})$; 作为超级电容器电 极材料, UCN-0.15-220-850电极在电流密度 $1.0 \mathrm{~A} \mathrm{~g}^{-1}$ 时的比电容为 $206 \mathrm{~F} \mathrm{~g}^{-1}$, 经过 5000 次循环充放电后其 比电容衰减率仅为 $2.4 \%$, 表现出优秀的电化学循环 稳定性. 此外, 当电流密度增加至 $50 \mathrm{~A} \mathrm{~g}^{-1}$ 时比电容 仍保持在 $135 \mathrm{~F} \mathrm{~g}^{-1}$, 表明其在超大电流密度下仍具有 良好的双电层电容特性. 超微孔碳纳米粒子 $\mathrm{UCN}-$ 0.15-220-850的优异的电化学性能来自于其规则的微 孔能够加速电解液离子的迁移, 提高其充放电能力; 丰富的堆积孔隙为电解液离子提供了缓冲区域, 减 小其扩散电阻; 大量的超微孔可以缩短电解液离子 到电极表面的传输距离, 从而大幅度提高电极材料 的比电容. 因此, 超微孔碳纳米粒子/微球 (UCNs/ $\mathrm{UCMs}$ ) 作为一种新型碳材料, 克服了传统微孔碳材 料不能在大电流下充放电的缺陷, 在超级电容器电
极材料方面必将展现出极其广阔的应用前景.

\section{4 功能化碳材料}

通常来说, 碳材料表面呈现疏水特性, 使得其在 水系电解液中有些孔无法或很难被电解液润湿，从 而降低了电化学性能. 因此在碳材料表面引人特殊 官能团或者杂原子(如 N, O, P, S, B等)已成为一种常 见的提高碳材料电化学性能的方法 ${ }^{[68 ~ 72]}$. 特殊官能 基团或者杂原子的引人可以在维持碳材料本身的优 良性能的基础上增加其亲水性、导电性和表面法拉第 反应位点. 在碳材料中引人氮元素可以实现碳材料 的氮功能化, 实现氮功能化主要有两种方法, 即原位 合成法和后处理法. 原位合成法是指通过碳化不同 的含氮前驱体制备含氮碳材料, 包括聚苯胺、聚吡 咯 ${ }^{[73]}$ 、聚丙烯腈 ${ }^{[74]}$ 、三聚氰胺类似物 ${ }^{[75]}$ 和含氮离子 液体 ${ }^{[76,77]}$ 等; 而后处理法是指通过氨、尿素或氮等离 子体 ${ }^{[78]}$ 处理多孔碳材料制备含氮碳材料. 在材料中 引人的氮元素分为两类: 化学氮和结构氮, 其中化学 氮主要以氨基和亚硝酰基等官能团的形式存在; 而 结构氮主要以吡咯型氮、嘧啶型氮和石墨型氮等形式 存在 ${ }^{[79]}$. 通常后处理法引人的是化学氮, 该法引人 的氮元素含量有限且分布不均匀, 其主要位于碳材 料的边缘或者缺陷上, 只能局部改变碳材料的性质, 而且在处理过程中会产生有毒气体, 对环境造成危 害. 而原位法引人的是结构氮, 该法直接利用含氮碳 源前驱体, 步骤更为简单, 可以在分子水平上对产物 的组成进行有效控制，使得氮元素均匀分布在材料 中, 从整体上提高材料的导电性和化学稳定性 ${ }^{[80]}$. 因 此，原位合成法是目前更为常用的氮功能化的方法. $\mathrm{Xu}$ 等人 ${ }^{[81]}$ 通过含氮有机盐的直接碳化制备掺氮多孔 碳材料, 所得碳材料的比表面积为 408 1171 $\mathrm{m}^{2} \mathrm{~g}^{-1}$,

(a) Phloroglucinol Terephthalalehyde
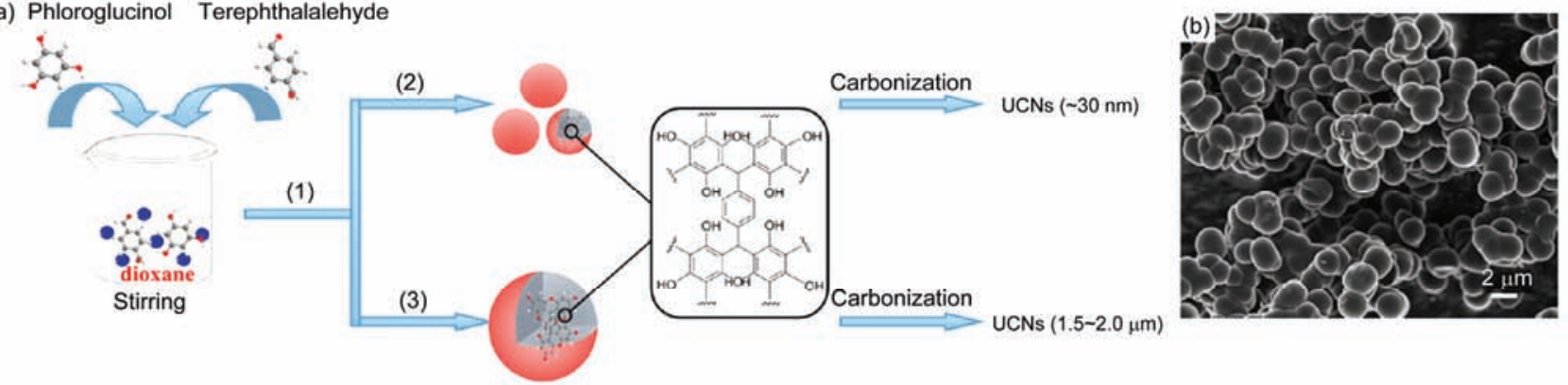

图 6 (网络版彩色)超微孔碳的合成过程示意图(a)和SEM图像(b) ${ }^{[67]}$

Figure 6 (Color online) (a) Schematic illustration of the formation process and (b) SEM images of ultramicroporous carbons ${ }^{[67]}$ 
含氮量为 $1.02 \%$ $8.59 \%$ (原子百分比), 作为超级电容 器电极材料时在电流密度为 $20 \mathrm{~A} \mathrm{~g}^{-1}$ 时的比电容高达 $188 \mathrm{~F} \mathrm{~g}^{-1}$. Sun等人 ${ }^{[82]}$ 选用葡萄糖为碳源、三聚氭胺为 氮源, 采用硬模板法直接合成含氮多孔石墨碳材料, 其比表面高达 $1027 \mathrm{~m}^{2} \mathrm{~g}^{-1}$, 含氮量为 $7.72 \%$ (质量百分 比), 在 $1 \mathrm{~A} \mathrm{~g}^{-1}$ 电流密度下的比电容为 $293 \mathrm{~F} \mathrm{~g}^{-1}$; 在有 机电解液中的能量密度和功率密度分别为 47.5 和 10.5 Wh k g ${ }^{-1}$.

本课题组 ${ }^{[83]}$ 首次选用间苯二胺为碳源和氮源, 利用间苯二胺和对苯二甲醛在醋酸催化作用下发生 席夫碱反应, 制备一种新型的含氮微孔有机聚合物, 经碳化后直接得到氮功能化的微孔碳纳米粒子 (N-MCNs). 图7(a)基于席夫碱反应的 N-MCNs合成过 程示意图. 典型样品 $\mathrm{N}-\mathrm{MCN}_{4,850}$ 展现出极高的比表面 积 $\left(1756 \mathrm{~m}^{2} \mathrm{~g}^{-1}\right)$ 、较大的孔体积 $\left(1.01 \mathrm{~cm}^{3} \mathrm{~g}^{-1}\right)$ 和丰富的 微孔分布(图7(b)); X射线光电子能谱(XPS)和元素分 析表明, 样品 $\mathrm{N}-\mathrm{MCN}_{4,850}$ 含有 $2.21 \%$ (质量百分数)的氮 元素; 作为超级电容器电极材料, 样品 $\mathrm{N}-\mathrm{MCN}_{4,850}$ 不 仅在低电流密度下具有极高的比电容(在 $0.1 \mathrm{~A} \mathrm{~g}^{-1}$ 电 流密度下比电容为 $391 \mathrm{~F} \mathrm{~g}^{-1}$ ), 而且在超高电流密度下 仍保持高的比电容, 例如在 $100 \mathrm{~A} \mathrm{~g}^{-1}$ 电流密度下比电 容为 $145 \mathrm{~F} \mathrm{~g}^{-1}$, 展现出超高倍率充放电性能. 经 5000 次循环充放电后其电容衰减率仅为 2\%(图7(c)), 因
此, 该氮功能化微孔碳纳米粒子可作为大容量高功 率密度超级电容器的理想电极材料.

本课题组 ${ }^{[84]}$ 还利用 1,5 -荎二胺为碳源和氮源, 在 过硫酸铵作用下合成了含氮碳微球 (NCMs), 合成过 程如图8所示. 典型的含氮碳微球样品 NCM-700的比 表面积为 $403 \mathrm{~m}^{2} \mathrm{~g}^{-1}$, 含氮量为 $5.94 \%$ (原子百分比), 微球直径为 $0.2 \sim 0.5 \mu \mathrm{m}$; 作为超级电容器电极材料, 在 $1 \mathrm{~A} \mathrm{~g}^{-1}$ 电流密度时的比电容为 $228 \mathrm{~F} \mathrm{~g}^{-1}$, 经 5000 次 充放电循环后比电容保持率为 $86 \%$, 展现出良好的 循环稳定性. 综上所述, 诸如以上两种原位氮功能化 的方法, 无需附加模板和后续化学活化, 操作简易可 行, 为制备满足实际应用需求的大容量高功率密度 超级电容器电极材料提供了可靠而有效的解决方案.

\section{5 多孔碳复合材料}

基于所用电极材料的不同, 超级电容器可分为 两种类型: (1) 高比表面积、惰性及导电材料. 这些材 料通过电荷在电极和电解液表面的聚集和分离进行 存储和释放能量如碳材料; (2) 通过材料表面上的快 速、可逆的氧化还原反应存储和释放能量(即赝电容) 的氧化还原活性材料, 如过渡金属氧化物. 与碳材料 相比, 金属氧化物可以为电化学超级电容器提供更 高的能量密度, 然而, 金属氧化物自身存在的弱电导

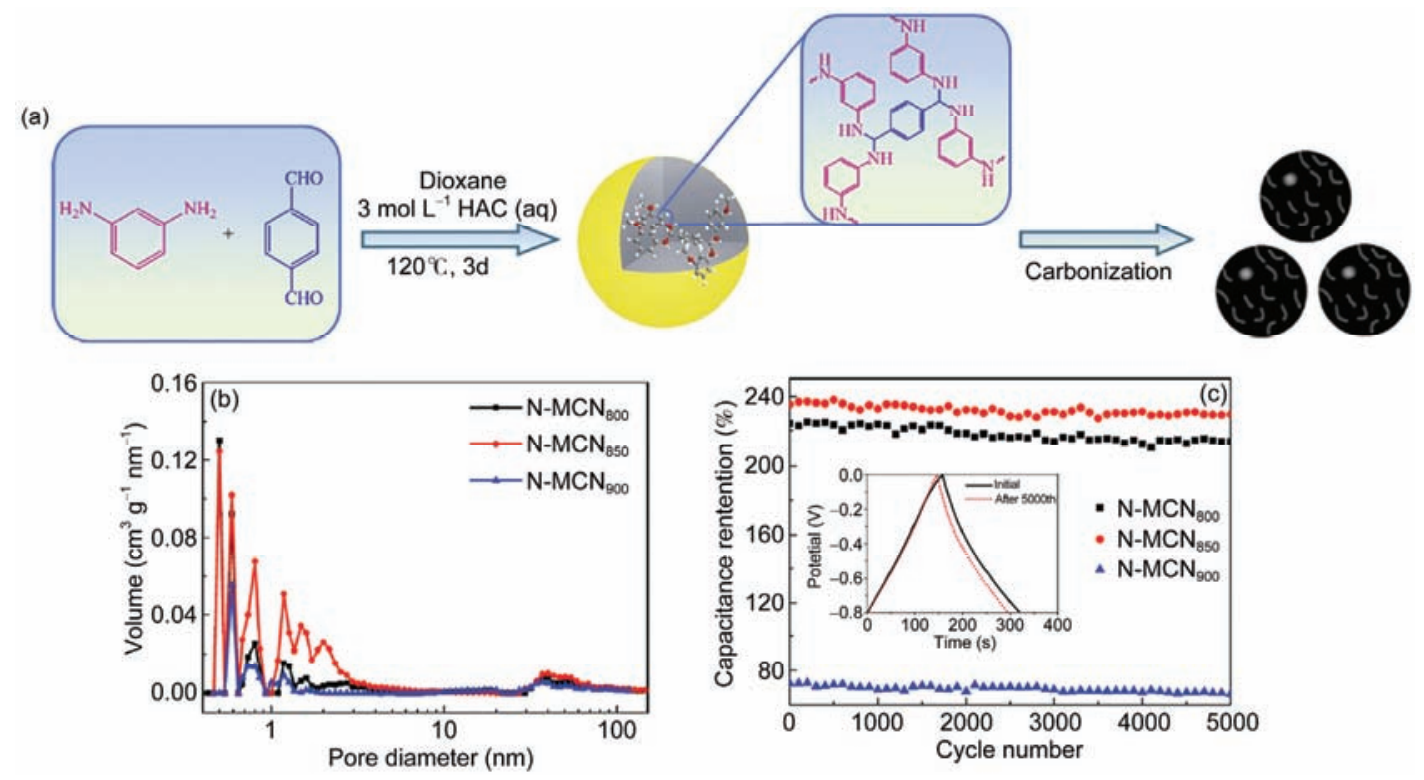

图 7 (网络版彩色)(a)基于席夫碱反应的N-MCNs合成过程示意图; (b) N-MCNs的孔径分布曲线; (c) N-MCNs电极在 $2.0 \mathrm{~A} \mathrm{~g}^{-1}$ 时的恒流充放电 曲线及其循环稳定性 ${ }^{[83]}$

Figure 7 (Color online) (a) Schematic illustration of the formation process. (b) Pore size distribution of N-MCNs. (c) Cycling stability of N-MCNs electrode at $2 \mathrm{~A} \mathrm{~g}^{-1}$ over 5000 cycles $^{[83]}$ 


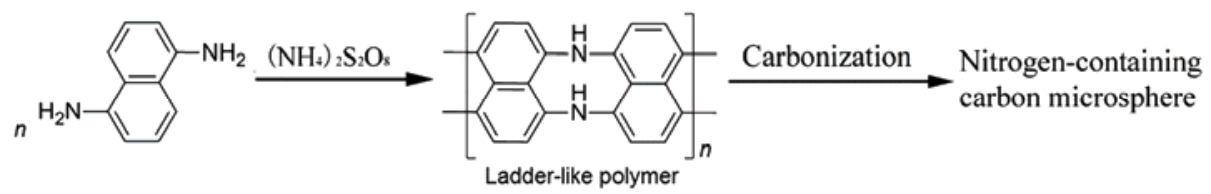

图 8 氮功能化碳微球的制备过程示意图 ${ }^{[84]}$

Figure 8 The schematic synthesis of nitrogen-containing carbon microspheres ${ }^{[84]}$

性使得其实际比电容远远小于其理论比电容. 为了 提高金属氧化物的比电容性能, 人们将研究重点集 中在导电材料与金属氧化物的复合材料的制备研究. 这类电极材料主要有两种制备方法: (1) 直接通过纳 米制造技术制备，即将金属氧化物薄膜直接涂层在 三维电导纳米材料上, 如金属 $\left(\mathrm{Ni}^{[85]}, \mathrm{Mn}^{[86]}\right)$ 、半导体

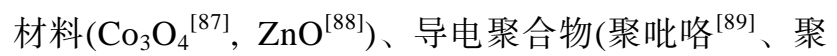
苯胺 ${ }^{[90]}$ 、聚噻吩 ${ }^{[1]}$ ) 和三维石墨烯 ${ }^{[75]}$. 虽然此类电极 材料能够表现出高比电容, 但制备工艺复杂, 活性材 料量低(通常低于 $0.5 \mathrm{mg} \mathrm{cm} \mathrm{cm}^{-2}$ ); (2) 将金属氧化物负 载在粉末碳材料上获得粉末电极材料. 这类粉末电 极材料中的活性材料的含量明显增加, 从而显著提 高了整个电极材料的能量密度. 结合工业化生产的 简易性, $\mathrm{MnO}_{2} / \mathrm{C}, \mathrm{NiO} / \mathrm{C}$ 等粉末电极材料有着广泛而 深远的应用前景. 近年来, 研究者都致力于 $\mathrm{MnO}_{2} / \mathrm{C}$, $\mathrm{NiO} / \mathrm{C}$ 复合材料的制备研究. $\mathrm{Lu}^{\text {等人 }}{ }^{[92]}$ 使用模板电化 学沉积法制备得到中空网络结构的三维 $\mathrm{NiO} / \mathrm{C}$ 复合 材料, 该复合材料作为超级电容器电极在 $1 \mathrm{~mol} \mathrm{~L}^{-1}$ $\mathrm{KOH}$ 电解液中, 电流密度为 $2.5 \mathrm{~A} \mathrm{~g}^{-1}$ 时的比电容值高 达572.5 F g ${ }^{-1}$, 但该方法步骤繁琐, 制备过程复杂. 本 课题组 ${ }^{[93]}$ 通过乳液-水热-活化及 $\mathrm{NiO}$ 负载方法制备负 载NiO的介孔碳微球复合材料Ni-AMCMs. Ni-AMCMs 表现出高比表面积 $\left(1096 \mathrm{~m}^{2} \mathrm{~g}^{-1}\right)$, 具有均一的介孔 (4.0 nm) 及 $0.5 ~ 1.0 \mu \mathrm{m}$ 的规则微球形貌(图9(a))等优 点. 当 $\mathrm{Ni}$ 源与 $\mathrm{AMCMs}$ 的质量比达 0.02 时, 制备的 $\mathrm{Ni}(0.02)-\mathrm{AMCMs}$ 作为超级电容器电极, 在 $6 \mathrm{~mol} \mathrm{~L}^{-1}$ $\mathrm{KOH}$ 电解液中, 电流密度为 $1.0 \mathrm{~A} \mathrm{~g}^{-1}$ 时的比电容达 $361 \mathrm{~F} \mathrm{~g}^{-1}$, 展现出相当好的电化学性能. 也有一些研 究者致力于 $\mathrm{MnO}_{2} / \mathrm{C}$ 复合材料的制备与性能研究, $\mathrm{Mao}$ 等人 ${ }^{[95]}$ 将不同形貌的纳米结构 $\mathrm{MnO}_{2}$ 负载在石墨 烯上得到 $\mathrm{MnO}_{2}(\mathrm{~N}-\mathrm{Mn}) / \mathrm{GTR}$ 复合材料. 其中, $\mathrm{N}-\mathrm{Mn} /$ GTR 13作超级电容器电极材料在 $0.1 \mathrm{~mol} \mathrm{~L}^{-1} \mathrm{~K}_{2} \mathrm{SO}_{4}$ 电 解液中, 电流密度为 $0.2 \mathrm{~A} \mathrm{~g} \mathrm{~g}^{-1}$ 时的比电容值高达 280 $\mathrm{F} \mathrm{g}^{-1}$. Feng等人 ${ }^{[96]}$ 使用一步水热法通过改变锰源的 含量制备得到新型花瓣状 $\mathrm{MnO}_{2}$ 纳米片/石墨烯复合
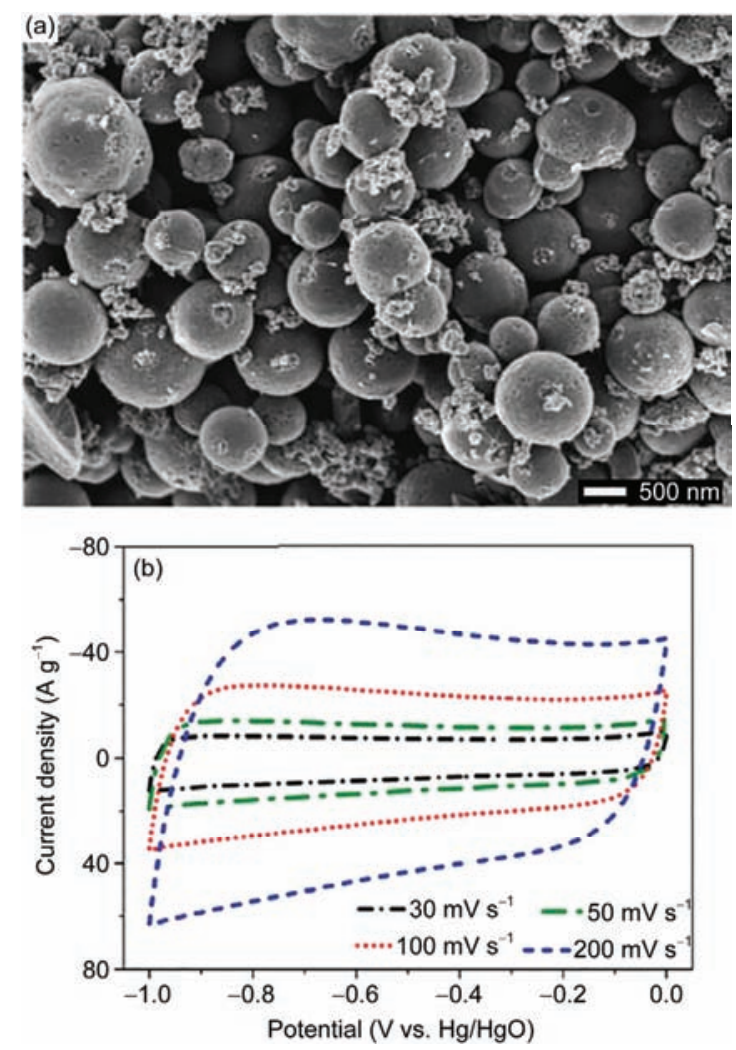

图 9 (网络版彩色)(a) $\mathrm{Ni} / \mathrm{C}$ 复合材料的SEM 图 ${ }^{[93]}$; (b) $\mathrm{MnO}_{2} / \mathrm{C}$ 的循环 伏安曲线 ${ }^{[94]}$

Figure 9 (Color online) (a) SEM images of Ni/C composite material ${ }^{[93]}$ and (b) $\mathrm{CV}$ curves of $\mathrm{MnO}_{2} / \mathrm{C}^{[94]}$

物, 该复合材料电极在 $1 \mathrm{~mol} \mathrm{~L}^{-1} \mathrm{Na}_{2} \mathrm{SO}_{4}$ 电解液中, 扫速为 $1.0 \mathrm{mV} \mathrm{s}^{-1}$ 时的比电容高达 $516.8 \mathrm{~F} \mathrm{~g}^{-1}$. Kiani等 人 $^{[97]}$ 将 $\mathrm{MnO}_{2}$ 负载在有序介孔碳(CMK-3)基质上制备 了 $\mathrm{MnO}_{2} / \mathrm{CMK}-3 . \mathrm{MnO}_{2} / \mathrm{CMK}-3$ 作为超级电容器电极 在 $0.5 \mathrm{~mol} \mathrm{~L}^{-1} \mathrm{Na}_{2} \mathrm{SO}_{4}$ 电解液中, 电流密度为 $1.0 \mathrm{~A} \mathrm{~g}^{-1}$ 时的比电容高达 $640 \mathrm{~F} \mathrm{~g} \mathrm{~g}^{-1}$. 近年来, 本课题组也制备 了金属氧化物负载碳材料的复合材料. 例如, 将 $\mathrm{MnO}_{2}$ 负载在部分石墨烯结构的多孔碳微球上得到 $\mathrm{MnO}_{2} / \mathrm{C}$ 复合材料 ${ }^{[94]}$. 首先, 通过水热-乳液聚合反应 和活化法制备的碳微球的孔洞中引人硝酸锰盐, 经 水热处理得到无定型 $\mathrm{MnO}_{2}$. 制得的 $\mathrm{MnO}_{2} / \mathrm{C}$ 的比表面 
积高达 $1135 \mathrm{~m}^{2} \mathrm{~g}^{-1}$, 且有规则球貌(粒径约 0.5 1.0 $\mu \mathrm{m})$, 这些都有利于离子的快速传输及快速充放电, 从而提高其作为超级电容器电极材料的电化学性能 (图9(b)). 当电流密度为 $1.0 \mathrm{~A} \mathrm{~g}^{-1}$ 时, $\mathrm{MnO}_{2} / \mathrm{C}$ 电极在 $6 \mathrm{~mol} \mathrm{~L}{ }^{-1} \mathrm{KOH}$ 电解液中的比电容达到 $459 \mathrm{~F} \mathrm{~g}^{-1}$.

此外, 还开展了多孔碳作为锂离子电池复合负极 材料的研究. 硅、锗在循环脱嵌锂过程中容易产生严 重的体积膨胀和机械损伤, 导致其循环性能很差 ${ }^{[98]}$, 而多孔碳具有良好的电子导电性和循环稳定性, 因此, 本课题组 ${ }^{[99]}$ 通过正硅酸乙酯的水解和缩聚反应, 反应 产物 $\mathrm{SiO}_{2}$ 与十六烷基三甲基溴化铵的静电作用, 再经 水热、碳化及镁热还原反应, 制备了介孔碳包覆 $\mathrm{Si}$ 纳 米粒子的 $\mathrm{Si} @ \mathrm{C}$ 介孔复合微球. 该微球作为锂离子电 池的负极材料, 在 $50 \mathrm{~mA} \mathrm{~g}$ 时经 100 次循环充放电后 的充电和放电比容量为 1038 和 $1053 \mathrm{mAh} \mathrm{g}^{-1}$, 在保持 高比电容量的同时, 大幅提高了其电化学循环稳定性. 制备的 $\mathrm{Ge} @ \mathrm{C}$ 介孔复合微球在 $50 \mathrm{~mA} \mathrm{~g}$ 时经100次循 环后的充电和放电比容量分别为 1077 和 $1099 \mathrm{mAh} \mathrm{g}^{-1}$, 表现出非常优异的循环充放电稳定性 ${ }^{[100]}$.

\section{2 结论与展望}

在各类能量存储器件中, 超级电容器是一种新 兴的绿色储能元件, 在便携式电子产品、电动汽车和 可再生能源等领域具有极其广阔的应用前景. 但是,
超级电容器的能量密度远远低于二次电池, 大幅限 制了其作为动力电源系统的应用. 电极材料是影响 超级电容器储能性能的关键因素, 超级电容器若要 同时满足高能量密度和功率密度的需求, 所用的电 极材料必须具有比电容高、比表面积大、导电率高、 循环寿命长和成本低等特点. 目前超级电容器最常 用的电极材料仍然是碳基材料, 而且活性碳基超级 电容器已经商业化, 但其无法满足脉冲大电流充放 电要求. 多孔碳具有制备简捷和成本低廉的优点, 容 易实现对其结构控制, 从而可以设计合成出高能量 密度和倍率性能以及长循环寿命的用于超级电容器 的多孔碳电极材料. 因此, 电化学性能高的低成本多 孔碳材料的设计与可控合成研究仍将是该领域一个 重要研究方向. 超级电容器电化学性能的影响因素 还有电解液和超级电容器结构等. 对于对称型超级 电容器, 具有大比表面积、可控孔径分布多孔碳材料 的宏量制备, 以及高分解电压电解液的开发尤为重 要; 非对称型超级电容器具有较高的能量密度, 特别 是锂离子混合型超级电容器, 其能量密度已接近锂 离子电池, 但其在大电流充放电过程中容量衰减比 较显著, 因此, 满足实际应用中对储能器件的高功率 和能量密度要求的非对称型超级电容器使用的新型 电极材料的制备和应用研究, 必将是能量存储与转 化领域中的又一个重要的研究方向.

\section{参考文献}

1 Simon P, Gogotsi Y, Dunn B. Where dobatteries end and supercapacitors begin? Science, 2014, 343: 1210-1211

2 Kötz R, Carlen M. Principles and applications of electrochemical capacitors. Electrochim Acta, 2000, 45: 2483-2498

3 Yang Z, Ren J, Zhang Z, et al. Recent advancement of nanostructured carbon for energy applications. Chem Rev, 2015, 115: 5159-5223

4 Wei W F, Cui X W, Chen W X, et al. Manganese oxide-based materials as electrochemical supercapacitor electrodes. Chem Soc Rev, 2011, 40: 1697-1721

5 Nam K W, Kim K B. A study of the preparation of $\mathrm{NiO}_{x}$ electrode via electrochemical route for supercapacitor applications and their charge storage mechanism. J Electrochem Soc, 2002, 149: 346-354

6 Miller J M, Dunn B, Tran T D, et al. Deposition of ruthenium nanoparticles on carbon aerogels for high energy density supercapacitor electrodes. J Electrochem Soc, 1997, 144: 309-311

7 Lee H Y, Goodenough J B. Ideal supercapacitor behavior of amorphous $\mathrm{V}_{2} \mathrm{O}_{5} \cdot n \mathrm{H}_{2} \mathrm{O}$ in potassium chloride (KCl) aqueous solution. J Solid State Chem, 1999, 148: 81-84

8 Sharma R K, Rastogi A C, Desu S B. Pulse polymerized polypyrrole electrodes for high energy density electrochemical supercapacitor. Electrochem Commun, 2008, 10: 268-272

9 Shown I, Ganguly A, Chen L C, et al. Conducting polymer-based flexible supercapacitor. Energy Sci Eng, 2015, 3: 2-26

10 Wang Y, Xia Y. Recent progress in supercapacitors: From materials design to system construction. Adv Mater, 2013, 25: 5336-5342

11 McCreery R L. Advanced carbon electrode materials for molecular electrochemistry. Chem Rev, 2008, 108: 2646-2687

12 Mayer S, Pekala R, Kaschmitter J. The aerocapacitor: An electrochemical double-layer energy-storage device. J Electrochem Soc, 1993, 140: 446-451 
13 Zhu J, Yang X, Fu Z, et al. Fabrication of ultra-low density, high surface area carbon aerogels and their application in supercapacitors. Mater Sci Forum, 2016, 852: 1349-1355

14 Futaba D N, Hata K, Yamada T, et al. Shape-engineerable and highly densely packed single-walled carbon nanotubes and their application as supercapacitor electrodes. Nat Mater, 2006, 5: 987-994

15 Zhang H, Cao G, Wang Z, et al. Tube-covering-tube nanostructured polyaniline/carbon nanotube array composite electrode with high capacitance and superior rate performance as well asgood cycling stability. Electrochem Commun, 2008, 10: 1056-1059

16 Huang Y, Liang J, Chen Y. An overview of the applications of graphene-based materials in supercapacitors. Small, 2012, 8: 1805-1834

17 Zhang K, Mao L, Zhang L L, et al. Surfactant-intercalated, chemically reduced graphene oxide for high performance supercapacitor electrodes. J Mater Chem, 2011, 21: 7302-7307

18 Zhao Y, Liu J, Hu Y, et al. Highly compression-tolerant supercapacitor based on polypyrrole-mediated graphene foam electrodes. Adv Mater, 2013, 25: 591-595

19 Gao W, Wan Y, Dou Y, et al. Synthesis of partially graphitic ordered mesoporous carbons with high surface areas. Adv Energy Mater, 2011, 1: 115-123

20 Yan Y, Cheng Q, Zhu Z, et al. Controlled synthesis of hierarchical polyaniline nanowires/ordered bimodal mesoporous carbon nanocomposites with high surface area for supercapacitor electrodes. J Power Sources, 2013, 240: 544-550

21 Ren J, Li L, Chen C, et al. Twisting carbon nanotube fibers for both wire-shaped micro-supercapacitor and micro-battery. Adv Mater, 2013, 25: 1155-1159

22 Lv T, Yao Y, Li N, et al. Highly stretchable supercapacitors based on aligned carbon nanotube/molybdenum disulfide composites. Angew Chem Int Ed, 2016, 55: 9191-9195

23 Li X, Zhao T, Wang K, et al. Directly drawing self-assembled, porous, and monolithic graphene fiber from chemical vapor deposition grown graphene film and its electrochemical properties. Langmuir, 2011, 27: 12164-12171

24 Pech D, Brunet M, Durou H, et al. Ultrahigh-power micrometre-sized supercapacitors based on onion-like carbon. Nat Nanotechnol, 2010, 5: 651-654

25 Chien H C, Cheng W Y, Wang Y H, et al. Ultrahigh specific capacitances for supercapacitors achieved by nickel cobaltite/carbon aerogel composites. Adv Funct Mater, 2012, 22: 5038-5043

26 Xie K, Qin X, Wang X, et al. Carbon nanocages as supercapacitor electrode materials. Adv Mater, 2012, 24: 347-352

27 Sun Y, Wu Q, Shi G. Graphene based new energy materials. Energy Environ Sci, 2011, 4: 1113-1132

28 Li Y, Fu Z Y, Su B L. Hierarchically structured porous materials for energy conversion and storage. Adv Funct Mater, 2012, 22: 4634-4667

29 Vu A, Qian Y, Stein A. Porous electrode materials for lithium-ion batteries-How to prepare them and what makes them special. Adv Energy Mater, 2012, 2: 1056-1085

30 Jiang H, Lee P S, Li C. 3D carbon based nanostructures for advanced supercapacitors. Energy Environ Sci, 2013, 6: 41-53

31 Wei S, Zhang H, Huang Y, et al. Pig bone derived hierarchical porous carbon and its enhanced cycling performance of lithium-sulfur batteries. Energy Environ Sci, 2011, 4: 736-740

32 Han Y, Dong X, Zhang C, et al. Easy synthesis of honeycomb hierarchical porous carbon and its capacitive performance. J Power Sources, 2013, 227: 118-122

33 Li H, Yuan D, Tang C, et al. Lignin-derived interconnected hierarchical porous carbon monolith with large areal/volumetric capacitances for supercapacitor. Carbon, 2016, 100: 151-157

34 Qie L, Chen W, Xu H, et al. Synthesis of functionalized 3D hierarchical porous carbon for high-performance supercapacitors. Energy Environ Sci, 2013, 6: 2497-2504

35 Tripathi P K, Liu M, Zhao Y, et al. Enlargement of uniform micropores in hierarchically ordered micro-mesoporous carbon for high level decontamination of bisphenol A. J Mater Chem A, 2014, 2: 8534-8544

36 Zhang P, Yuan J, Fellinger T P, et al. Improving hydrothermal carbonization by using poly(ionic liquid)s. Angew Chem Int Ed, 2013, 52: 6028-6032

37 Qian D, Lei C, Hao G P, et al. Synthesis of hierarchical porous carbon monoliths with incorporated metal-organic frameworks for enhancing volumetric based $\mathrm{CO}_{2}$ capture capability. ACS Appl Mater Interfaces, 2012, 4: 6125-6132

38 Dutta S, Bhaumik A, Wu K C W. Hierarchically porous carbon derived from polymers and biomass: Effect of interconnected pores on energy applications. Energy Environ Sci, 2014, 7: 3574-3592

39 Lv Y, Gan L, Liu M, et al. A self-template synthesis of hierarchical porous carbon foams based on banana peel for supercapacitor electrodes. J Power Sources, 2012, 209: 152-157

40 Wickramaratne N P, Xu J, Wang M, et al. Nitrogen enriched porous carbon spheres: Attractive materials for supercapacitor electrodes 
and $\mathrm{CO}_{2}$ adsorption. Chem Mater, 2014, 26: 2820-2828

41 Guo C X, Li C M. A self-assembled hierarchical nanostructure comprising carbon spheres and graphene nanosheets for enhanced supercapacitor performance. Energy Environ Sci, 2011, 4: 4504-4507

42 Zhou D D, Liu H J, Wang Y G, et al. Ordered mesoporous/microporous carbon sphere arrays derived from chlorination of mesoporous TiC/C composite and their application for supercapacitors. J Mater Chem, 2012, 22: 1937-1943

43 Fan Y, Liu P F, Huang Z Y, et al. Porous hollow carbon spheres for electrode material of supercapacitors and support material of dendritic Pt electrocatalyst. J Power Sources, 2015, 280: 30-38

44 Lei Z, Christov N, Zhang L L, et al. Mesoporous carbon nanospheres with an excellent electrocapacitive performance. J Mater Chem, 2011, 21: 2274-2281

45 Choma J, Jamioła D, Augustynek K, et al. New opportunities in Stöber synthesis: Preparation of microporous and mesoporous carbon spheres. J Mater Chem, 2012, 22: 12636-12642

$46 \mathrm{Xu} \mathrm{Z,} \mathrm{Guo} \mathrm{Q.} \mathrm{A} \mathrm{simple} \mathrm{method} \mathrm{to} \mathrm{prepare} \mathrm{monodisperse} \mathrm{and} \mathrm{size-tunable} \mathrm{carbon} \mathrm{nanospheres} \mathrm{from} \mathrm{phenolic} \mathrm{resin.} \mathrm{Carbon,} \mathrm{2013,} \mathrm{52:}$ 464-467

47 Choma J, Fahrenholz W, Jamioła D, et al. Development of mesoporosity in carbon spheres obtained by Stöber method. Micropor Mesopor Mat, 2014, 185: 197-203

48 Fang Y, Gu D, Zou Y, et al. A low-concentration hydrothermal synthesis of biocompatible ordered mesoporous carbon nanospheres with tunable and uniform size. Angew Chem Int Ed, 2010, 49: 7987-7991

49 Qiao Z A, Guo B, Binder A J, et al. Controlled synthesis of mesoporous carbon nanostructures via a "silica-assisted" strategy. Nano Lett, 2012, 13: 207-212

50 Li Q, Jiang R, Dou Y, et al. Synthesis of mesoporous carbon spheres with a hierarchical pore structure for the electrochemical doublelayer capacitor. Carbon, 2011, 49: 1248-1257

51 Xiong W, Liu M, Gan L, et al. A novel synthesis of mesoporous carbon microspheres for supercapacitor electrodes. J Power Sources, 2011, 196: 10461-10464

52 Qian J, Liu M, Gan L, et al. A seeded synthetic strategy for uniform polymer and carbon nanospheres with tunable sizes for high performance electrochemical energy storage. Chem Commun, 2013, 49: 3043-3045

53 Ma X, Liu M, Gan L, et al. Synthesis of micro-and mesoporous carbon spheres for supercapacitor electrode. J Solid State Electr, 2013, 17: $2293-2301$

54 Ma X, Gan L, Liu M, et al. Mesoporous size controllable carbon microspheres and their electrochemical performances for supercapacitor electrodes. J Mater Chem A, 2014, 2: 8407-8415

55 Liu M, Qian J, Zhao Y, et al. Core-shell ultramicroporous@microporous carbon nanospheres as advanced supercapacitor electrodes. J Mater Chem A, 2015, 3: 11517-11526

56 Xu S, Liang L, Li B, et al. Research progress on microporous organic polymers. Prog Chem, 2011, 23: 2085-2094

57 Li B, Guan Z, Yang X, et al. Multifunctional microporous organic polymers. J Mater Chem A, 2014, 2: 11930-11939

58 Dawson R, Stöckel E, Holst J R, et al. Microporous organic polymers for carbon dioxide capture. Energy Environ Sci, 2011, 4: $4239-4245$

59 Jin Y, Voss B A, Jin A, et al. Highly $\mathrm{CO}_{2}$-selective organic molecular cages: What determines the $\mathrm{CO}_{2}$ selectivity. J Am Chem Soc, 2011, 133: 6650-6658

$60 \mathrm{Wu} \mathrm{H}$, Thibault C G, Wang H, et al. Effect of temperature on hydrogen and carbon dioxide adsorption hysteresis in an ultramicroporous MOF. Micropor Mesopor Mat, 2016, 219: 186-189

61 Sekizkardes A K. An ultra-microporous organic polymer for high performance carbon dioxide capture and separation. Chem Commun, 2015, 51: 13393-13396

62 Li X, Wei B. Supercapacitors based on nanostructured carbon. Nano Energy, 2013, 2: 159-173

63 Dubal D, Ayyad O, Ruiz V, et al. Hybrid energy storage: The merging of battery and supercapacitor chemistries. Chem Soc Rev, 2015, 44: $1777-1790$

64 Wang G, Zhang L, Zhang J. A review of electrode materials for electrochemical supercapacitors. Chem Soc Rev, 2012, 41: 797-828

65 Lorrmann V, Reichenauer G, Weber C, et al. Electrochemical double-layer charging of ultramicroporous synthetic carbons in aqueous electrolytes. Electrochim Acta, 2012, 86: 232-240

66 Xu B, Hou S, Duan H, et al. Ultramicroporous carbon as electrode material for supercapacitors. J Power Sources, 2013, 228: 193-197

67 Zhao Y, Liu M, Gan L, et al. Ultramicroporous carbon nanoparticles for the high-performance electrical double-layer capacitor electrode. Energy Fuel, 2014, 28: 1561-1568

68 Kurniawan A, Ong L, Kurniawan F, et al. Easy approach to synthesize N/P/K co-doped porous carbon microfibers from cane molasses as 
a high performance supercapacitor electrode material. RSC Adv, 2014, 4: 34739-34750

69 Luo H, Liu Z, Chao L, et al. Synthesis of hierarchical porous N-doped sandwich-type carbon composites as high-performance supercapacitor electrodes. J Mater Chem A, 2015, 3: 3667-3675

70 Si W, Zhou J, Zhang S, et al. Tunable N-doped or dual N, S-doped activated hydrothermal carbons derived from human hair and glucose for supercapacitor applications. Electrochim Acta, 2013, 107: 397-405

71 Ramasahayam S K, Nasini U B, Shaikh A U, et al. Novel tannin-based Si, P co-doped carbon for supercapacitor applications. J. Power Sources, 2015, 275: 835-844

72 Ling Z, Wang G, Zhang M, et al. Boric acid-mediated B,N-codoped chitosan-derived porous carbons with a high surface area and greatly improved supercapacitor performance. Nanoscale, 2015, 7: 5120-5125

73 Ghenaatian H, Mousavi M, Rahmanifar M. High performance hybrid supercapacitor based on twonanostructured conducting polymers: Self-doped polyaniline and polypyrrole nanofibers. Electrochim Acta, 2012, 78: 212-222

74 Zhong M, Kim E K, McGann J P, et al. Electrochemically active nitrogen-enriched nanocarbons with well-defined morphology synthesized by pyrolysis of self-assembled block copolymer. J Am Chem Soc, 2012, 134: 14846-14857

75 Wen Z, Wang X, Mao S, et al. Crumpled nitrogen-doped graphene nanosheets with ultrahigh pore volume for high-performance supercapacitor. Adv Mater, 2012, 24: 5610-5616

76 Guo D C, Mi J, Hao G P, et al. Ionic liquid $\mathrm{C}_{16} \mathrm{mimBF}_{4}$ assisted synthesis of poly(benzoxazine-co-resol)-based hierarchically porous carbons with superior performance in supercapacitors. Energy Environ Sci, 2013, 6: 652-659

77 Zhang S, Mandai T, Ueno K, et al. Hydrogen-bonding supramolecular protic salt as an "all-in-one" precursor for nitrogen-doped mesoporous carbons for $\mathrm{CO}_{2}$ adsorption. Nano Energy, 2015, 13: 376-386

78 Jeong H M, Lee J W, Shin W H, et al. Nitrogen-doped graphene for high-performance ultracapacitors and the importance of nitrogen-doped sites at basal planes. Nano Lett, 2011, 11: 2472-2477

79 Wood K N, O’Hayre R, Pylypenko S. Recent progress on nitrogen/carbon structures designed for use in energy and sustainability applications. Energy Environ Sci, 2014, 7: 1212-1249

80 Shen W, Fan W. Nitrogen-containing porous carbons: synthesis and application. J Mater Chem A, 2013, 1: 999-1013

$81 \mathrm{Xu} \mathrm{B}$, Zheng D, Jia M, et al. Nitrogen-doped porous carbon simply prepared by pyrolyzing a nitrogen-containing organic salt for supercapacitors. Electrochim Acta, 2013, 98: 176-182

82 Sun L, Tian C, Fu Y, et al. Nitrogen-doped porous graphitic carbon as an excellent electrode material for advanced supercapacitors Chem Eur J, 2014, 20: 564-574

83 Zhao Y, Liu M, Deng X, et al. Nitrogen-functionalized microporous carbon nanoparticles for high performance supercapacitor electrode. Electrochim Acta, 2015, 153: 448-455

84 Zhu D, Wang Y, Gan L, et al. Nitrogen-containing carbon microspheres for supercapacitor electrodes. Electrochim Acta, 2015, 158: $166-174$

85 Ho C L, Wu M S. Manganese oxide nanowires grown on ordered macroporous conductive nickel scaffold for high-performance supercapacitors. J Phys Chem C, 2011, 115: 22068-22074

86 Li Q, Wang Z L, Li G R, et al. Design and synthesis of $\mathrm{MnO}_{2} / \mathrm{Mn} / \mathrm{MnO}_{2}$ sandwich-structured nanotube arrays with high supercapacitive performance for electrochemical energy storage. Nano Lett, 2012, 12: 3803-3807

87 Liu J, Jiang J, Cheng C, et al. $\mathrm{Co}_{3} \mathrm{O}_{4}$ nanowire@ $\mathrm{MnO}_{2}$ ultrathin nanosheet core/shell arrays: A new class of high-performance pseudocapacitive materials. Adv Mater, 2011, 23: 2076-2081

88 He Y B, Li G R, Wang Z L, et al. Single-crystal ZnO nanorod/amorphous and nanoporous metal oxide shell composites: Controllable electrochemical synthesis and enhanced supercapacitor performances. Energy Environ Sci, 2011, 4: 1288-1292

89 Wang J G, Yang Y, Huang Z H, et al. Rational synthesis of $\mathrm{MnO}_{2}$ /conducting polypyrrole@carbon nanofiber triaxial nano-cables for high-performance supercapacitors. J Mater Chem, 2012, 22: 16943-16949

90 Jiang $\mathrm{H}$, Ma J, Li C. Polyaniline- $\mathrm{MnO}_{2}$ coaxial nanofiber with hierarchical structure for high-performance supercapacitors. J Mater Chem, 2012, 22: 16939-16942

91 Liu R, Lee S B. $\mathrm{MnO}_{2}$ /poly(3,4-ethylenedioxythiophene) coaxial nanowires by one-step coelectrodeposition for electrochemical energy storage. J Am Chem Soc, 2008, 130: 2942-2943

92 Lu X F, Lin J, Huang Z X, et al. Three-dimensional nickel oxide@ carbon hollow hybrid networks with enhanced performance for electrochemical energy storage. Electrochim Acta, 2015, 161: 236-244

93 Liu M, Gan L, Xiong W, et al. Nickel-doped activated mesoporous carbon microspheres with partially graphitic structure for supercapacitors. Energy Fuel, 2013, 27: 1168-1173

94 Liu M, Gan L, Xiong W, et al. Development of $\mathrm{MnO}_{2}$ /porous carbon microspheres with a partially graphitic structure for high performance 
supercapacitor electrodes. J Mater Chem A, 2014, 2: 2555-2562

95 Mao L, Zhang K, Chan H S O, et al. Nanostructured $\mathrm{MnO}_{2}$ /graphene composites for supercapacitor electrodes: The effect of morphology, crystallinity and composition. J Mater Chem, 2012, 22: 1845-1851

96 Feng X, Yan Z, Chen N, et al. The synthesis of shape-controlled $\mathrm{MnO}_{2} /$ graphene composites via a facile one-step hydrothermal method and their application in supercapacitors. J Mater Chem A, 2013, 1: 12818-12825

97 Kiani M, Khani H, Mohammadi N. $\mathrm{MnO}_{2}$ /ordered mesoporous carbon nanocomposite for electrochemical supercapacitor. J Solid State Electr, 2014, 18: 1117-1125

98 Zhou L, Zhao D, Lou X W. Double-shelled CoMn ${ }_{2} \mathrm{O}_{4}$ hollow microcubes as high-capacity anodes for lithium-ion batteries. Adv Mater, 2012, 24: 745-748

99 Ma X, Liu M, Gan L, et al. Novel mesoporous Si@C microspheres as anodes for lithium-ion batteries. Phys Chem Chem Phys, 2014, 16: 4135-4142

100 Liu M, Ma X, Gan L, et al. A facile synthesis of a novel mesoporous Ge@C sphere anode with stable and high capacity for lithium ion batteries. J Mater Chem A, 2014, 2: 17107-17114 


\title{
Porous carbon materials: Design, synthesis and applications in energy storage and conversion devices
}

\author{
LIU MingXian, MIAO Ling, LU WenJing, ZHU DaZhang, XU ZiJie, GAN LiHua* \& CHEN LongWu \\ Shanghai Key Lab of Chemical Assessment and Sustainability, School of Chemical Science and Engineering, Tongji University, Shanghai 200092, \\ China \\ * Corresponding author, E-mail: ganlh@tongji.edu.cn
}

The dramatic environmental pollution and energy shortages have spurred internationally unprecedented interest in developing new energy technologies. Supercapacitors have emerged as a new class of green electrochemical devices for energy conversion and storage and are promising candidates for extensive applications. As a key component of supercapacitors, electrode materials are a crucial factor to the electrochemical performance based on its properties including surface area, pore structure, conductivity and surface functionalization. The well-designed synthesis strategies and conditions are usually fatal to tailor four mentioned properties. Due to the advantages of low cost, high specific surface area and conductivity, controllable microstructure, easy surface functionalization, remarkable chemical stability and outstanding electrolyte ion accessibility, porous carbon materials tailored through well-designed synthesis strategies and conditions, exhibit high energy density and power density as well as superb electrochemical cycling stability. In this review, we firstly provide a brief description of energy storage mechanisms for different types of electrode materials, followed by a comprehensive overview of recent advances in development of different carbon-based materials with activated carbon, carbon aerogels, carbon fiber, mesoporous carbon, carbon nanotube and graphene. Then we state the key parameters to evaluate the electrochemical properties, such as specific capacitance, energy density and power density, and also discuss the relationship between the influence parameters (e.g. surface area, pore structure, conductivity, and surface properties) and enhanced performances. Further, according to the research work of our group, we present a summary on the design, synthesis and applications in energy conversion and storage based on porous carbon materials, including carbons with different pore distributions (hierarchical porous carbon, porous carbon sphere, ultramicroporous carbon), functionalized porous carbon and porous carbon composite materials. In terms of carbons with different pore distributions, we list some characteristic synthetic methods (e.g. the self-template strategy for banana-peel-derived hierarchical porous carbon foams, the seeded synthetic strategy for phenolic-resin-derived porous carbon nanospheres and the solvothermal method for phloroglucinol-terephthaldehyde-derived ultramicroporous carbon nanoparticles), which can be concluded that micropores (especially ultramicropores) are electrochemically available for electrolyte ions because the solvation shell is squeezed through the pores less than the solvated ion size and such distortion reduces distance between the electrode surface and the ion center, while mesopores offer highly efficient pore channels for ion penetration and transport. In terms of functionalized porous carbon, we adopt the in situ synthesis approach to prepare nitrogen-doped carbons (e.g. poly(1, 5-diaminonapthalene)-derived nitrogen-containing carbon microspheres and phenylenediamine-terephthalaldehyde-derived nitrogen-functionalized microporous carbon nanoparticles), which demonstrate that heteroatom doping, on the one hand, increases the surface wettability in the aqueous electrolyte to improve the mass transfer efficiency, and on the other hand, endows additional psedocapacitance for the electrode. In terms of porous carbon composite materials, we combine carbon-based materials with pseudocapacitive metal oxides (e.g. $\mathrm{NiO}$ and $\mathrm{MnO}_{2}$ ) for achieving high-performance supercapacitors, which is a wise choice to increase the energy density without sacrificing the high power capability. These strategies and methods provide new ideas to simple and highly efficient design of porous carbon materials and may be extendable to other systems such as metal or metal oxide materials. Additionally, the future trend of carbon based electrode materials for energy conversion and storage device is discussed. There are extensive applications outside the area of high-rate electrochemical energy storage, such as drug delivery, photonic crystals, adsorption and separation, and catalysis.

porous carbon, synthesis, energy-storage material, supercapacitor, electrochemical performance 